\title{
Faecalibacterium prausnitzii prevents physiological damages in a chronic low-grade inflammation murine model
}

\author{
Rebeca Martín ${ }^{1,2,3}$, Sylvie Miquel ${ }^{1,2}$, Florian Chain ${ }^{1,2}$, Jane M Natividad ${ }^{3}$, Jennifer Jury ${ }^{3}$, Jun Lư ${ }^{3}$, Harry Sokol ${ }^{1,2,4,5}$, \\ Vassilia Theodorou ${ }^{6}$, Premysl Bercik ${ }^{3}$, Elena F Verdu ${ }^{3}$, Philippe Langella ${ }^{1,2,3^{*}}$ and Luis G Bermúdez-Humarán ${ }^{1,2^{*}}$
}

\begin{abstract}
Background: The human gut houses one of the most complex and abundant ecosystems composed of up to $10^{13}-10^{14}$ microorganisms. The importance of this intestinal microbiota is highlighted when a disruption of the intestinal ecosystem equilibrium appears (a phenomenon called dysbiosis) leading to an illness status, such as inflammatory bowel diseases (IBD). Indeed, the reduction of the commensal bacterium Faecalibacterium prausnitzii (one of the most prevalent intestinal bacterial species in healthy adults) has been correlated with several diseases, including IBD, and most importantly, it has been shown that this bacterium has anti-inflammatory and protective effects in pre-clinical models of colitis. Some dysbiosis disorders are characterized by functional and physiological alterations. Here, we report the beneficial effects of F. prausnitzii in the physiological changes induced by a chronic low-grade inflammation in a murine model. Chronic low-grade inflammation and gut dysfunction were induced in mice by two episodes of dinitro-benzene sulfonic acid (DNBS) instillations. Markers of inflammation, gut permeability, colonic serotonin and cytokine levels were studied. The effects of F. prausnitzii strain A2-165 and its culture supernatant (SN) were then investigated.
\end{abstract}

Results: No significant differences were observed in classical inflammation markers confirming that inflammation was subclinical. However, gut permeability, colonic serotonin levels and the colonic levels of the cytokines IL-6, INF- $\gamma$, IL-4 and IL-22 were higher in DNBS-treated than in untreated mice. Importantly, mice treated with either F. prausnitzii or its SN exhibited significant decreases in intestinal permeability, tissue cytokines and serotonin levels.

Conclusions: Our results show that F. prausnitzii and its SN had beneficial effects on intestinal epithelial barrier impairment in a chronic low-grade inflammation model. These observations confirm the potential of this bacterium as a novel probiotic treatment in the management of gut dysfunction and low-grade inflammation.

Keywords: Microbiota, Dysbiosis, IBD-remission, Low-grade inflammation, Probiotics

\section{Background}

The human gastrointestinal tract (GIT) is one of the most complex ecosystems. The interaction between GIT microbiota and the host is indispensable for the maturation of the immune system and for the regulation of intestinal physiology [1]. Firmicutes (which encompasses Clostridum leptum and C. coccoides) and Bacteroidetes are the two dominant phyla in the human gut, representing $90 \%$ of the

\footnotetext{
* Correspondence: philippe.langella@jouy.inra.fr; luis.bermudez@jouy.inra.fr 'INRA, Commensal and Probiotics-Host Interactions Laboratory, UMR 1319 Micalis, F-78350 Jouy-en-Josas, France

Full list of author information is available at the end of the article
}

microbiota, with Actinobacteria being the third, comprising of only $3 \%[2]$.

When the normal microbial ecosystem is disrupted, different non-predominant bacteria can bloom, constituting a potential trigger for disease. The term dysbiosis refers to a type of microbial imbalance that may promote disease. The strongest relationship between dysbiosis and pathological condition has been observed in inflammatory bowel diseases (IBD), where the proportion of Firmicutes is lower than in healthy subjects [3,4]. IBD, composed mainly of ulcerative colitis (UC) and Crohn's disease $(\mathrm{CD})$, are characterized by an unusual 
activation of the GIT-immune system leading to a chronic inflammation of the gut with a mix of remission and exacerbation periods. It has recently been suggested that the colonic microbiota is also abnormal in patients with irritable bowel syndrome (IBS), with a 2-fold increased ratio of the Firmicutes to Bacteroidetes [5]. IBS is a symptom-based diagnosis characterized by chronic abdominal pain and altered bowel habits [6] in the absence of detectable structural abnormalities [7].

IBD and IBS are classically considered as dichotomous conditions. However, increasing evidence, such as the involvement of brain-gut axis dysfunction [8-10], as well as abnormal microbiota, supports some pathogenetic overlap between the two conditions [8]. Furthermore, the identification of low-grade inflammation [9], histopathological observations and mucosal cytokine levels in the colon of a subset of IBS patients $[10,11]$ and the presence of IBS-like symptoms in quiescent IBD [8,12-14] support this hypothesis.

Low-grade inflammation can persist in many IBD patients who have otherwise obtained clinical remission [15], as well as barrier alterations due to functional impairment of tight junction proteins [16]. In fact, impaired gut barrier function appears to precede the development of IBD [17] and increased epithelial permeability causes mucosal immune activation predisposing or enhancing disease progression [18].

Due to the importance of the microbiota in the development of these dysbiosis-related diseases, there is currently increasing interest in the development of microbiota-modulating therapies, including the use of probiotics $[19,20]$. F prausnitzii is a candidate probiotic bacterium that has been proposed as a sensor of intestinal health [21]. Changes in the abundance of this commensal anti-inflammatory bacterium [22] and major member of the Clostridium leptum group [23] have been linked to dysbiosis in several human disorders [21], such as IBD, where a low abundance was found in patients exhibiting endoscopic recurrence 6 months after surgery [3]. Furthermore, the recovery of $F$. prausnitzii counts after relapse is associated with maintenance of clinical remission in UC patients [24]. Besides, F. prausnitzii has shown protective effects in inflammation parameters in both acute [22] and chronic [25] models of chemical-induced active inflammation; however its beneficial role during low-grade inflammation and gut dysfunction has not been tested yet. For these reasons, we hypothesized a possible protective role of $F$. pausnitzii on gut dysfunction mediated by low-grade inflammation. Therefore, we investigated the potential of $F$. prausnitzii as a probiotic for gut dysfunction prevention and therapy. We tested the effects of $F$. prausnitzii in a murine model of chronic low-grade inflammation mimicking some of the most common gut dysfunction parameters found in IBD patients under remission.

\section{Results \\ Validation of a low-grade inflammation model in DNBS-treated mice}

The model of low-grade DNBS inflammation developed in this study involves the induction of a low grade, subclinical inflammatory status, followed by a recovery period and a reactivation period mimicking the relapsing nature in IBD (Figure 1A). The challenge with a subcolitic DNBS dose (i.e. second DNBS administration) did not induce important changes in weight or colonic macroscopic or histological scores confirming the absence of a strong inflammation (Figure 1B, C, E, G, H). The degree of infiltration by polymorphonuclear neutrophils, as measured by tissue MPO activity, as well as Lipocalin-2 (Lcn-2), an early inflammation marker in inflammatory and autoimmune disorders, did not differ significantly between DNBS-treated and control animals (Figure 1D, F). These observations suggest that DNBStreated mice tend to display a low-grade inflammatory status rather than a strong inflammation (as observed in IBD patients) thereby validating our model for further analysis of physiological changes induced by this chronic low-grade, subclinical inflammation. The findings for all these markers thus suggest that the colons of DNBStreated mice have a low-grade inflammatory status. Comparison of the levels of these markers with those previously published by our group following a colitic dose of DNBS that induced severe or moderate chronic inflammation [25] provided further evidence that this protocol leads to lowgrade inflammatory conditions (Figure 2).

\section{Faecalibacterium prausnitzii A2-165 and its SN counteract DNBS-induced hyperpermeability in vivo}

The gut of DNBS-treated mice showed high permeability to the paracellular tracer FITC-dextran in vivo (KruskalWallis test followed by a Dunn's Multiple Comparison test, $p<0.05$ ) (Figure 3A). We tested whether F. prausnitzii modulates this DNBS-induced gut dysfunction. Treatment with either F. prausnitzii or its SN resulted in a significant reduction of intestinal permeability (Kruskal-Wallis test followed by a Dunn's Multiple Comparison test, $p<0.05$ ) (Figure 3A). To confirm that these findings corresponded to local paracellular translocation (i.e. in the colon) of the FITC-dextran in vivo in this model, intestinal permeability was assessed in vitro in colonic samples mounted in Ussing chambers extracted from mice at sacrifice (Figure 3B). The results were consistent with the pattern observed in vivo (Kruskal-Wallis test followed by a Dunn's Multiple Comparison test, $p<0.05)$ confirming local translocation of the FITC-dextran. We then tested F. prausnitzii SN (which had a large effect on intestinal permeability in vivo) with samples from DNBS-challenged mice by Ussing chamber analysis 


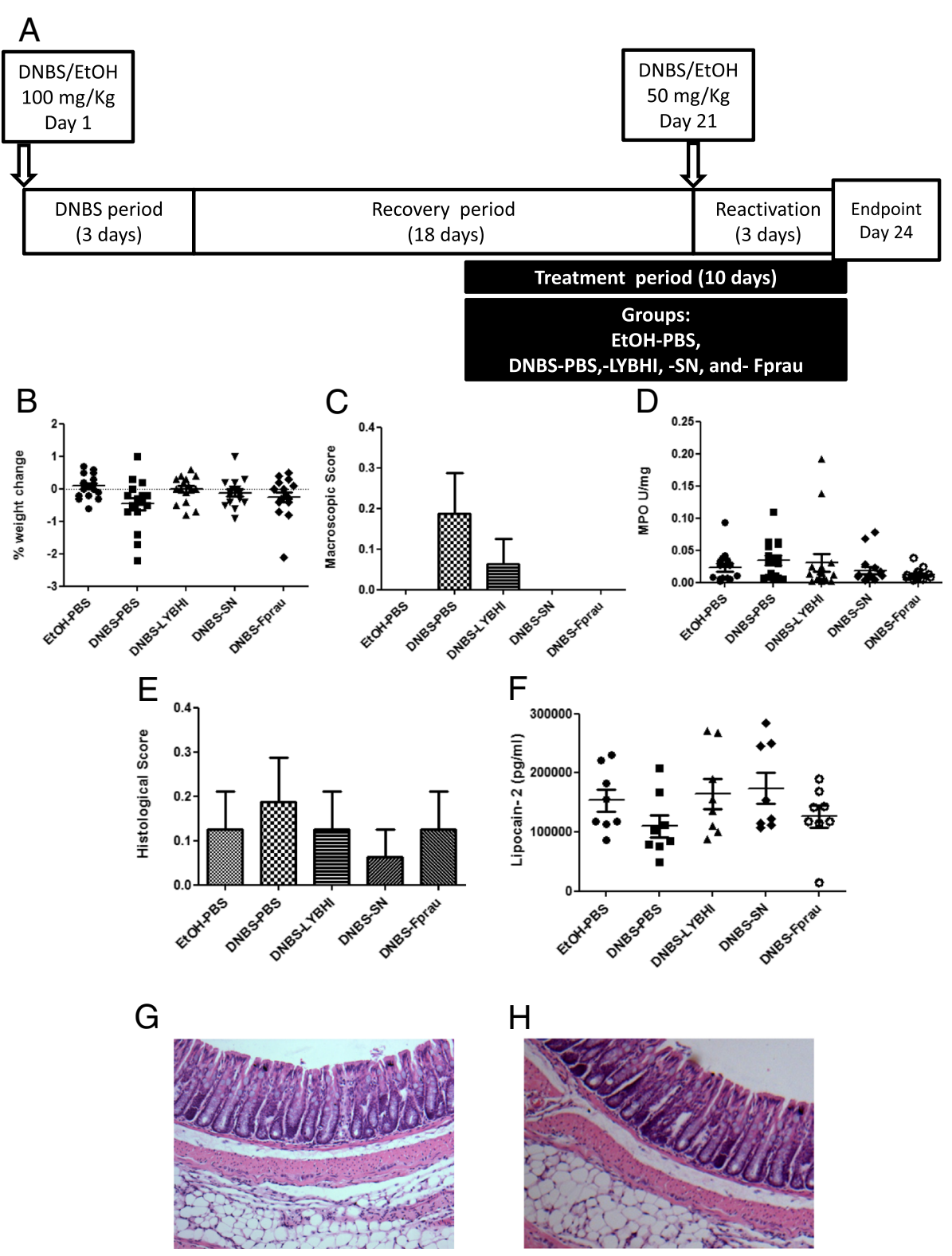

Figure 1 Experimental protocols for the mouse model of chronic micro-inflammation and absence of inflammation. A) Colitis was induced by intrarectal administration of $100 \mathrm{mg} / \mathrm{Kg}$ of DNBS in solution in 30\% ethanol (EtOH). Control mice (without colitis) received only $30 \%$ $\mathrm{EtOH}$. The effects of DNBS are highest during the first 3 days after its administration (DNBS period). Ten days after the end of the DNBS period, bacterial culture, PBS, or supernatant (SN) were intragastrically administered daily for 10 days (gavage period). Colitis was reactivated 21 days after the first DNBS injection with a second injection of $50 \mathrm{mg} / \mathrm{Kg}$ of DNBS solution. Three days after reactivation mice were sacrificed. The severity of the reactivated colitis was assessed from difference in body weight (B), macroscopic score (C), MPO activity (D), histological score (E) $(n=16$ mice per group) and lipocalin-2 levels (F) $\left(n=2^{*} 4=8\right.$ mice per group) between control non-inflamed (EtOH-PBS), control inflamed (DNBS-PBS), bacteria-free culture medium (DNBS-LYBHI), F. prausnitzii strain A2-165 (DNBS-Fprau), and F. prausnitzii A2-165 SN (DNBS-SN) groups. Experiments were performed at least in duplicate. Normal appearance of the colon of a control mouse with no inflammation (G) and of a mouse with micro-inflammation $\mathbf{( H )}$.

(Figure 3B): the ${ }^{51}$ Cr-EDTA flux, measured in \% Hot Sample $/ \mathrm{hr} / \mathrm{cm}^{2}$, was lower with SN than PBS (KruskalWallis test followed by a Dunn's Multiple Comparison test, $p<0.05$ ) (Figure 3B). No significant differences were obtained in tissue conductance $\left(\mathrm{mS} / \mathrm{cm}^{2}\right)$ (Figure 3C).

\section{F. prausnitzii restores DNBS-induced apical junction proteins decrease}

These effects on gut permeability prompted us to investigate the involvement of epithelial adherens junction (AJ) and tight junction (TJ) proteins, by assaying for relevant mRNA levels. The mRNA levels for Claudin-4 (Kruskal- 

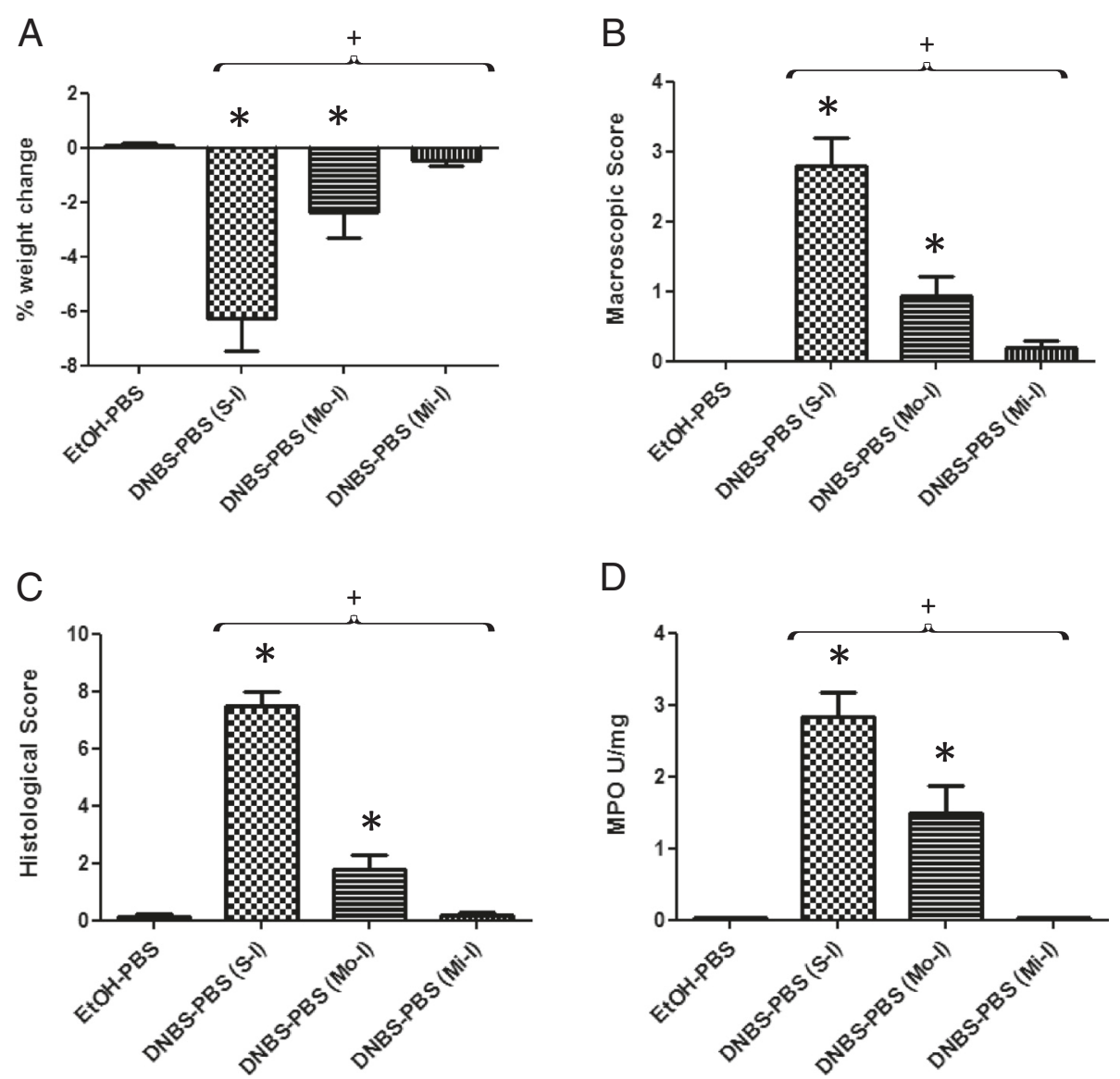

Figure 2 Comparison of inflammation measures in severe and moderate colitis models [25] with the micro-inflammation model employed in this study. Severity of the colitis was assessed from differences in body weight (A), macroscopic score (B), histological score (C) and MPO activity (D) between the control non-inflamed (EtOH-PBS), control inflamed severe colitis model (DNBS-PBS (S-I)) [25], moderate colitis model (DNBS-PBS (M-I)) [25] and micro-inflammation model (DNBS-PBS (Mi-I)). Experiments were performed at least in duplicate. Comparisons involved the non-parametric Kruskal-Wallis test was used followed by a Dunn's Multiple Comparison test. ${ }^{*} p<0.05$ vs. DNBS-PBS, $+p<0.05$ ( $n=16$ mice per group).

Wallis test,$p=0.0006$ ), E-cadherin (Kruskal-Wallis test, $p=0.0175$ ), F11r (JAM1) (Kruskal-Wallis test, $p=0.0023$ ), Occludin (Kruskal-Wallis test, $p=0.0170$ ) and ZO-1 (Kruskal-Wallis test, $p=0.0001$ ) were all less abundant in DNBS-treated mice than in control mice (Kruskal-Wallis test followed by a Dunn's Multiple Comparison test, $p<0.05$ ) (Figure 4); Claudin-1, 2, 5 and 15 mRNA levels were unaffected (data no shown). F. prausnitzii and its SN tended to normalize the amounts of all these mRNA transcripts, the only statistically significant regulation was found for Claudin-4 and F11r (Kruskal-Wallis test followed by a Dunn's Multiple Comparison test, $p<0.05$ ) (Figure 4). Claudin-4, E-cadherin, F11r and Occludin expression was also analyzed by immunofluorescence (Additional file 1: Figure S1). Staining for the four proteins was reduced in $\mathrm{DNBS}+\mathrm{PBS}$ mice compared to $\mathrm{EtOH}+\mathrm{PBS}$ group (Additional file 1: Figure S1). According to the qPCR results, $F$. prausnitzii tends to increase the expression of all the proteins.
A systemic and mucosal reduction in pro-inflammatory cytokine production correlates with the protective effects of $F$. prausnitzii and its SN after DNBS challenge

We investigated the cytokines involved in this low-grade inflammation model and the mechanism by which $F$. prausnitzii and its SN protect the intestinal mucosa. We assayed 13 cytokines (including those involved in the Th1, Th2, Th17 and Th22 pathways) in both colonic and serum samples after the second DNBS injection (Figure 5). The concentrations of IL-13, IL-1 $\alpha$, IL-6, IL-22, IL-2, IL-27, IL4 , IFN- $\gamma$, and TNF- $\alpha$ in colon samples were higher in DNBS-treated mice than in controls (Figure 5A and data not shown), consistent with local inflammation. Similarly, cytokine concentrations in serum samples were consistent with the low-grade inflammatory status (Figure 5B and data not shown). Strikingly, the concentration of IL-6, IFN- $\gamma$ and IL- 4 in colon samples and of IL- 6 and IL-22 in serum was statistically significantly reduced by treatment with either F. prausnitzii or its SN (Kruskal-Wallis test followed 

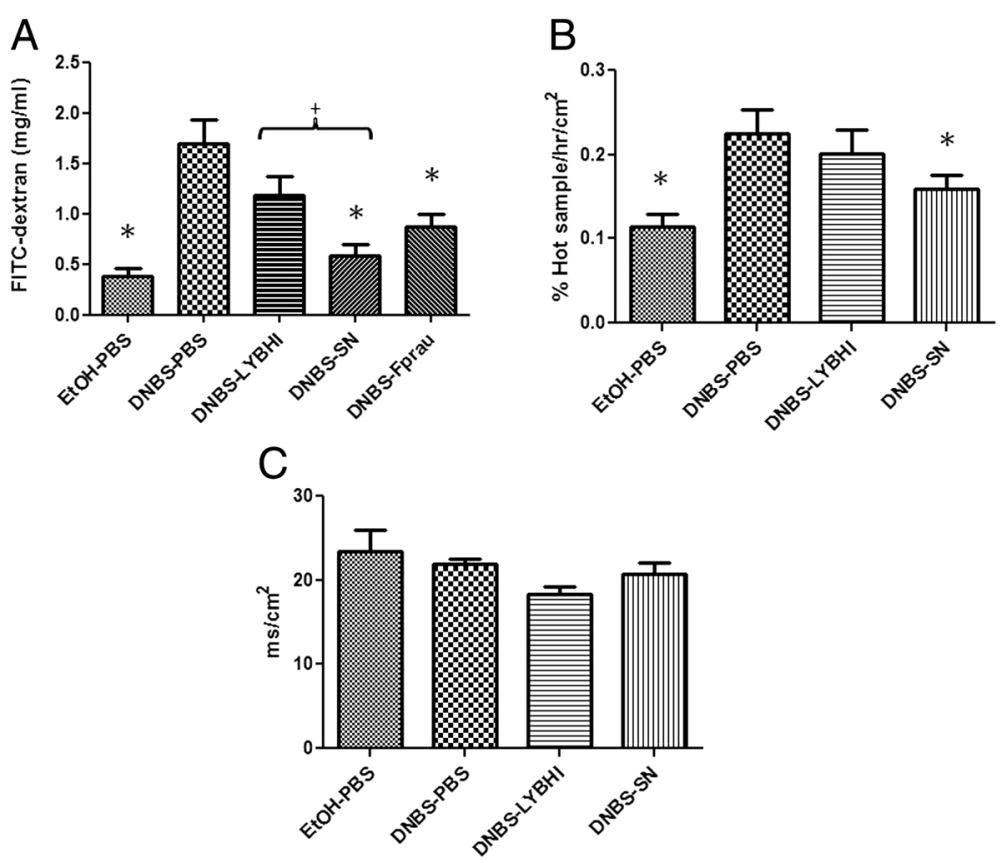

Figure 3 In vivo and in vitro permeability measurements. For in vivo measurements of gut permeability, animals were orally gavaged with FITC-dextran (A) ( $n=16$ mice per group). For in vitro measures, sections of colon were mounted in Ussing chambers and ${ }^{51} \mathrm{Cr}$-EDTA flux (\% Hot Sample $\left./ \mathrm{hr} / \mathrm{cm}^{2}\right)$ (B) and tissue conductance $\left(\mathrm{mS} / \mathrm{cm}^{2}\right) \mathbf{( C )}$ were measured ( $n=6$ mice per group). Control non-inflamed (EtOH-PBS), control inflamed (DNBS-PBS), bacteria-free culture medium (DNBS-LYBHI), F. prausnitzii strain A2-165 (DNBS-Fprau), and F. prausnitzii A2-165 SN (DNBS-SN) groups. Experiments were performed at least in duplicate. Comparisons involved the non-parametric Kruskal-Wallis test was used followed by a Dunn's Multiple Comparison test. ${ }^{*} p<0.05$ vs. DNBS-PBS, $+p<0.05$.

by a Dunn's Multiple Comparison test, $p<0.05$ ) and there were clear tendencies for these treatments to reduce colonic IL-22 and serum IL-13 concentrations (Figure 5).

\section{F. prausnitzii and its SN restore serotonin variations observed in DNBS-treated mice}

Serotonin (also called 5-HT for 5-hydroxyptamine) of the key neurotransmitters in the GIT, and affects motility, sensation and secretion [26]. We therefore studied serotonin concentrations in colon tissue and content by ELISA. Both colonic (Figure 6A) and colon content (Figure 6B) serotonin concentrations were higher in DNBS-treated mice than EtOH-treated controls (KruskalWallis test followed by a Dunn's Multiple Comparison test, $p<0.05)$. Treatments with both $F$. prausnitzii and its SN counteracted these increases (Kruskal-Wallis test followed by a Dunn's Multiple Comparison test, $p<0.05$ ) (Figure 6A, B). Serotonin reuptake transporter (SERT) mRNA was assayed by qPCR in colon tissues: the value for inflamed mice was half than for control non-inflamed mice $\left(2^{-\Delta \Delta C t}=0.49\right)$; the value for mice treated with $F$. prausnitzii was the same as that for normal healthy mice $\left(2^{-\Delta \Delta \mathrm{Ct}}=1.09\right.$ vs. EtOH-PBS). Similar positive effects were found in SN-treated mice $\left(2^{-\Delta \Delta C t}=1.29 v s\right.$. EtOH-PBS) although the culture medium (LYBHI) itself had a positive effect $\left(2^{-\Delta \Delta C t}=0.98 v s\right.$. EtOH-PBS $)$.

\section{Discussion}

There is growing evidence pointing at the role of the gut microbiota in the development of IBD $[3,4]$. Furthermore, at least $40 \%$ of IBD patients in clinical remission have ongoing histological evidence of inflammation [15] and suffer from altered gut functions with the involvement of brain-gut axis dysfunction [8,26,27]. Interestingly, previous transient or chronic inflammation can lead to persistent gut dysfunction [28] and it has been suggested that the host microbiota may affect the two-ways communication pathway between the gut and the brain [29].

Because the microbiota is a determinant of normal gut function and immunity [30], and because there is evidence of dysbiosis in IBD, there is increasing interest in the development of new adjuvant therapies based on modulation of the microbiota [31]. The traditional probiotic approach based on lactic acid bacteria (LAB) strains can be now extended to new candidate beneficial bacteria from the intestinal microbiota, including $F$. prausnitzii. This metabolic active species is a member of the C. leptum group, and is one of the most prevalent commensal bacteria in the human gut [2], involved in colonic epithelial physiology [32], and a reduction in its abundance has been observed in IBD patients [24]. The anti-inflammatory properties of the F. prausnitzii 


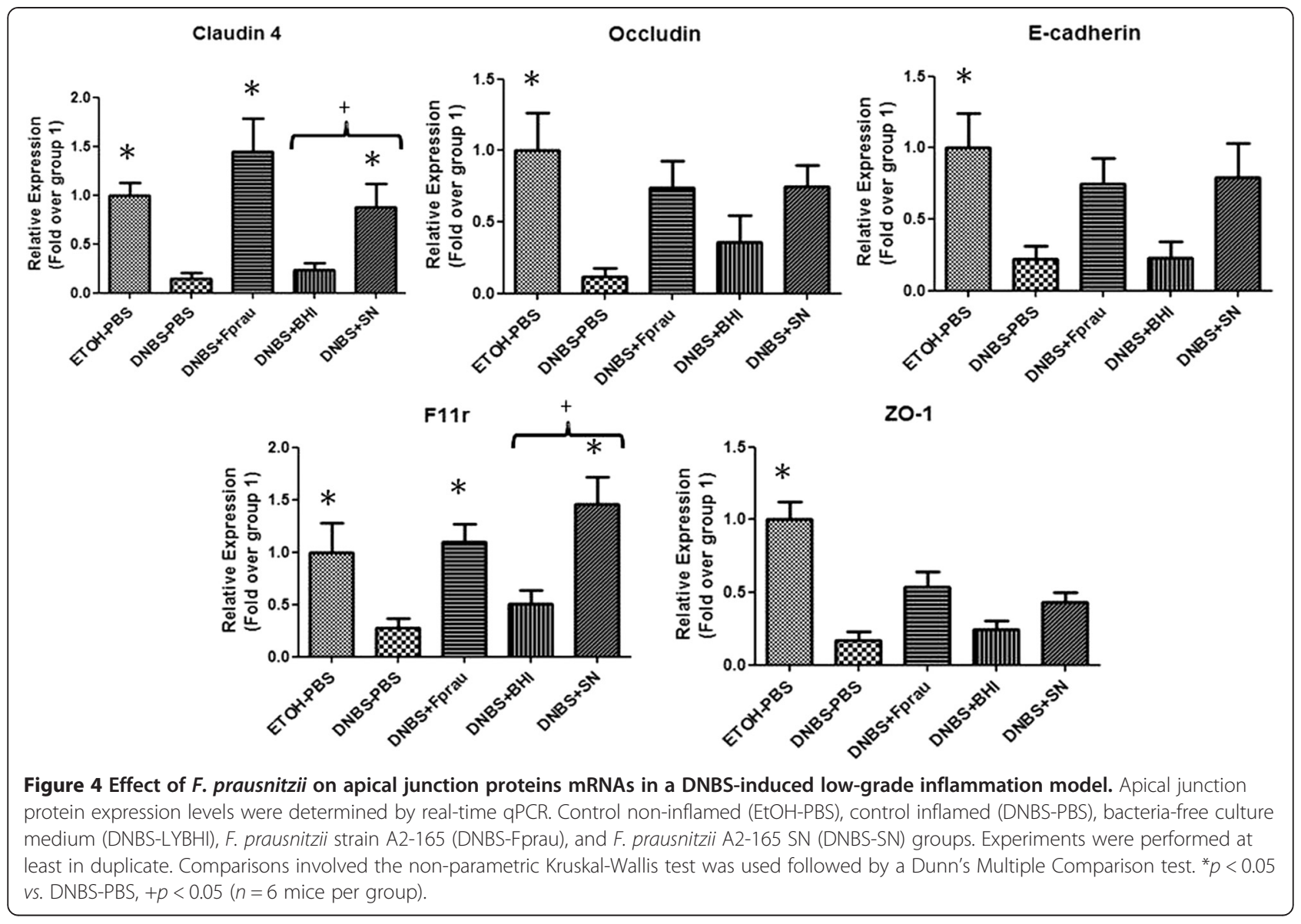

reference strain A2-165 have been demonstrated in vitro in various cellular models and validated in vivo in murine models of TNBS-induced acute colitis [22] and DNBS-induced chronic severe and moderate colitis [25].

We investigated the beneficial effects of both $F$. prausnitzii and its filtered SN (with the consequent loss of high molecular weight molecules) using a chronic low-grade inflammatory model, which mimics gut dysfunction related to colonic low-grade inflammation $[11,12]$. We adjusted the dose of DNBS such that the challenge induced low-grade inflammation, as it has been described for TNBS [33], a model that resembles DNBS [34]. To mimic the relapsing character of IBD and reach a remission status, we used a chronic protocol with recovery and reactivation periods. Our results show that chronic, low-dose administration of DNBS did not induce severe colonic inflammation: there was no macro- or microscopic damages or granulocyte infiltration. Furthermore, no increase in the levels of lipocalin 2 (Lcn-2), an early marker of inflammation, was observed [35]. Lcn-2 is an innate immune protein produced by a variety of cells, and its levels are increased during several pathologic conditions, including immune complexmediated inflammatory or autoimmune disorders $[36,37]$.
These various results indicate the absence of any substantial active inflammatory tone in this DNBStreated mouse model. However, the presence of a low-grade inflammation was confirmed by the small increases in some pro-inflammatory cytokine titers (including IL- 6 and INF- $\gamma$ ). The levels of most of the cytokines investigated, mainly in serum, were low, with no statistically significant difference between groups. Nevertheless, serum IL-13, colonic IFN- $\gamma$ and IL-4, and both serum and colonic IL- 6 and IL-22 were higher in DNBS treated mice. IFN- $\gamma$ is a Th1 cytokine and an important activator of macrophages; it is involved in the regulation of inflammation and in barrier dysfunction through the redistribution and expression of TJ proteins [38]. The pro-inflammatory Th1 cytokine IL-6 modulates the excitability of submucosal neurons and increases colonic secretory function. The cytokine IL-22 regulates various processes linked to several autoimmune disorders [39] exerting both antimicrobial and pro-inflammatory functions. IL-4 is a Th-2 cytokine, canonical of IL-13 [40], with stimulatory effects on B- and T-cells [41]; as well as involvement in dichotomous regulation of Th cell differentiation, IL-4 induces so-called alternative macrophage activation [42]. IL-4 and IL-13 can decrease cellular 


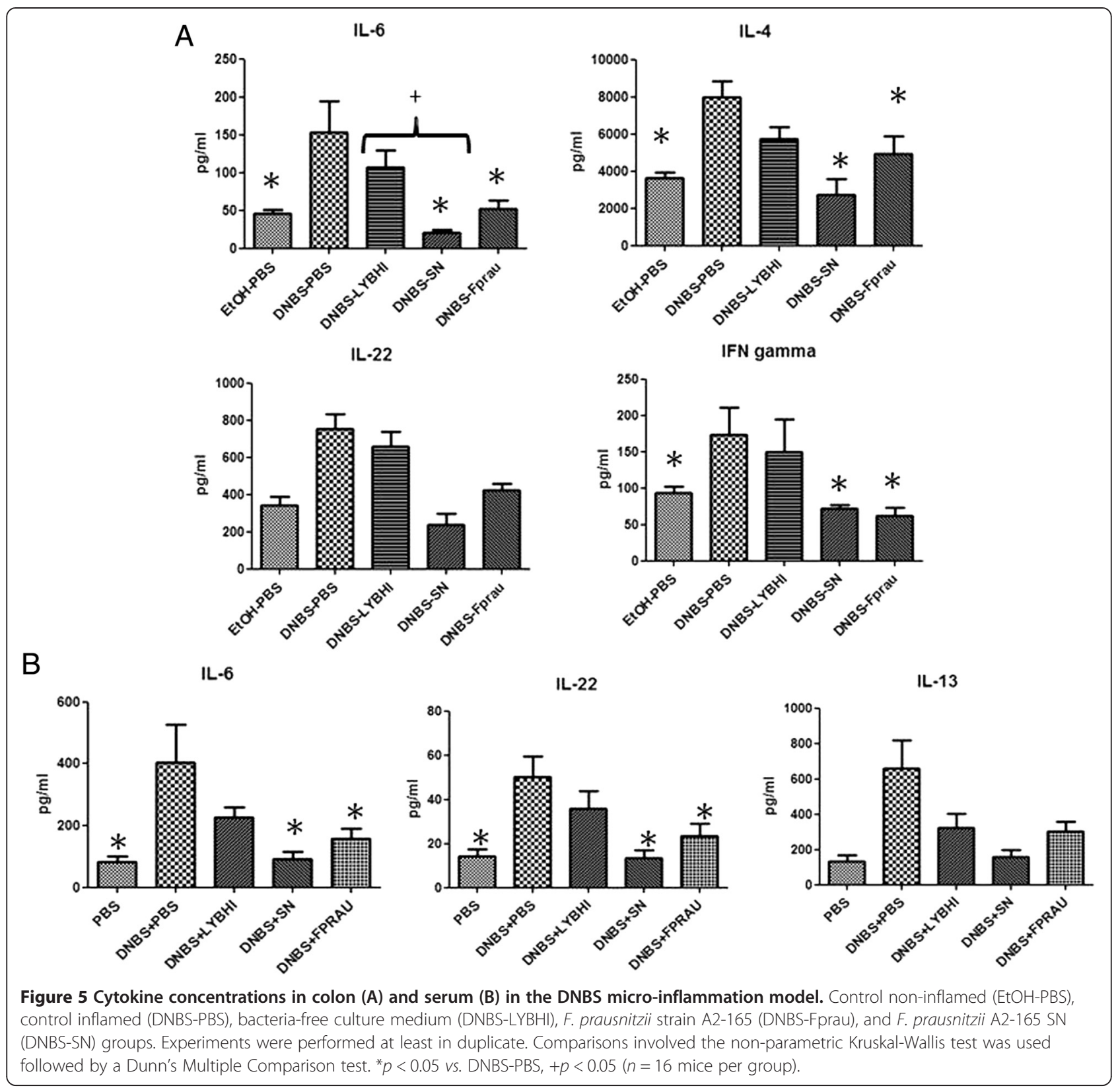

trans-epithelial electric resistance (TEER) in vitro and in vivo $[43,44]$. Our findings for the cytokines are difficult to interpret as they seem to involve both Th-1 and Th-2 immune responses as well as effects in the Th-17/Th-22 subset. This is not surprising because the particular inflammatory environment of our model could be the consequence of cross-regulation between all these possible pathways [11]. Although the profile of cytokines after chronic low dose DNBS challenge is not completely characterized, the altered cytokine profiles we document here in DNBS-treated mice and untreated control mice confirm mild immunological dysfunctions in the model. Furthermore, both $F$. prausnitzii and SN treatments had beneficial effects on indicators of immune status.

As IBD patients in remission often exhibit gut dysfunction [45], we therefore chose to study colonic permeability and serotonin levels as functional markers in our model. Treatment with either F. prausnitzzi or its SN normalized in vivo permeability, suggesting that overall permeability was improved. SN also improved permeability in vitro suggesting that the SN acts to protect the paracellular pathway in DNBS-challenged mice. This result is consistent with the findings by Carlssonn et al. that $F$. prausnitzii SN counteracted the negative effects of DSS challenge on colonic permeability [46]. 

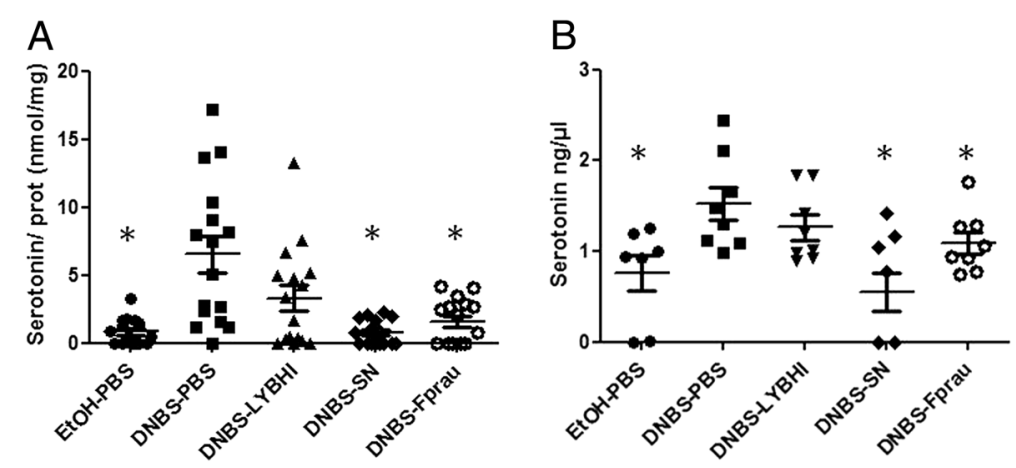

Figure 6 Serotonin concentrations in colon (A) and colon contents (B) in the DNBS micro-inflammation model. Control non-inflamed (EtOH-PBS), control inflamed (DNBS-PBS), bacteria-free culture medium (DNBS-LYBHI), F. prausnitzii strain A2-165 (DNBS-Fprau), and F. prausnitzii A2-165 SN (DNBS-SN) groups. Experiments were performed at least in duplicate.Comparisons involved the non-parametric Kruskal-Wallis test was used followed by a Dunn's Multiple Comparison test. ${ }^{*} p<0.05$ vs. DNBS-PBS, $+p<0.05$ ( $n=16$ mice per group for analysis of colon samples and $n=8$ mice per group for analysis of colon content samples).

Increased permeability after reactivation was accompanied by a reduction in the expression of apical junction proteins. Treatment with either F. prausnitzii or its SN restored the levels of Claudin- 4 and the junctional adhesion molecule F11r (JAM1). Overall, our results suggest that the protective effect of $F$. prausnitzii and its SN may be the consequence, at least in part, of an effect on tight junctions mediated through certain tight junction proteins. Other common biomarkers in gut dysfunction are related to gut dysmotility [47]. Although serotonin is not a direct marker of motility, it stimulates peristalsis, secretion, vasodilatation and sensory signaling in the gut [48]. It also activates the immune cells to produce pro-inflammatory mediators [49] being a link between inflammation and functional symptoms. Enterochromaffin cells (EC) containing serotonin transduce chemical and mechanical stimuli from the intestinal lumen by releasing the serotonin [50]. Serotonin selective reuptake transporter (SERT), expressed on intestinal enterocytes, terminates the action of 5 -HT by eliminating it from the interstitial space [48]. Dysfunctional mucosal serotonin signaling has been implicated in visceral sensitivity and altered motility in IBD patients [50,51]. Colonic levels of 5-HT were high in DNBS-challenged mice, both in colon tissue and content. This result is in agreement with previous studies reporting altered levels of this hormone in murine models of TNBS-induced inflammation and low grade inflammation [33]. Both $F$. prausnitzii and its $\mathrm{SN}$ were able to restore the serotonin level to normal. In T-cell-mediated models of inflammation, an increase in EC and a decrease SERT function have been described [52]. The cytokines IFN- $\gamma$ and IL13 (restored in both F. prausnitzii- and SN-treated mice) are involved in the metabolism of 5-HT, IFN- $\gamma$ decreases SERT expression in human epithelial Caco-2 cells in vitro [53], and IL-13 contributes to increasing EC numbers [52]. In agreement with what has been described for TNBS [54], we found lower SERT levels in DNBS-challenged mice. Moreover, F. prausnitzii and its SN restored these levels to normal. However, it is possible that the abundance of SERT reflects the general status of the epithelium, and it is not necessarily a direct consequence of the actions of $F$ prausnitizii or its SN. Indeed, the LYBHI medium may have an effect on SERT. This is not the first time that culture media (LYBHI or MRS) have been reported to show intrinsic anti-inflammatory properties in vivo and in vitro [25]; this is presumably due to some of the components of the medium, as previously observed with MRS culture medium [55].

\section{Conclusion}

Chronic low-grade inflammation is common in patients with IBD in clinical remission as well as in IBS patients $[15,28]$. Patients with histological inflammation at baseline are at increased risk of clinical relapse, hospitalization, surgery, and colon cancer in observational longitudinal studies [15]. It is widely recognized that microbiota can impact on gut homeostasis. Our study proposes that $F$. prausnitzii, one of the major members of the gut microbiota, protects from gut dysfunction-related symptoms in animal models. In summary, we describe novel protective effects of both $F$. prausnitzii and its $\mathrm{SN}$ on chronic low-grade inflammation, in a murine model mimicking the relapsing low-grade inflammatory conditions present in some IBD patients under remission. This bacterium exerts different beneficial effects in terms of cytokine levels, gut permeability and serotonin pathway. Furthermore, our results suggest that the protective effect of $F$. prausnitzii and its SN may be the consequence, at least in part, of barrier enhancement through certain tight junction proteins. These results provide further evidence of the potential of $F$. prausnitzii as a novel probiotic for gut-dysfunction management. Future studies will need to be performed to validate the described beneficial effect in patients gut dysfunction and low grade 
inflammation. Also, it will be important to determine whether disease associated dysbiosis is corrected, or whether the effect is independent of a modulation of the gut microbiota.

\section{Methods}

Bacterial strains and growth conditions

Faecalibacterium prausnitzii strain A2-165 (DSMZ collection, Braunschweig, Germany) (DSM N ${ }^{\circ} 17677$ ) was grown in LYBHI medium (brain-heart infusion medium supplemented with $0.5 \%$ yeast extract) (Difco, Detroit, USA) supplemented with $1 \mathrm{mg} / \mathrm{ml}$ cellobiose (SigmaAldrich Chemie GmbH, Buchs, Switzerland), $1 \mathrm{mg} / \mathrm{ml}$ maltose (Sigma-Aldrich), and $0.5 \mathrm{mg} / \mathrm{ml}$ cysteine (Sigma-Aldrich) at $37^{\circ} \mathrm{C}$ in an anaerobic chamber as previously described [22]. The supernatant of F. prausnitzii cultures $(\mathrm{SN})$ were recovered by centrifugation filtered through $0.45 \mu \mathrm{m}$ pore-size filters (VWR, Haasrode, Belgium) and stored at $-80 \mathrm{C}$ until use.

\section{Animals}

Male C57BL/6 mice (6-8 weeks old) (Janvier, Le Genest Saint Isle, France or Taconic mice New York, USA) were maintained either at the animal care facilities of the National Institute of Agricultural Research (IERP, INRA, Jouy-en-Josas, France) or at the animal care facilities of McMaster University (CAF, Hamilton, Ontario, Canada) under specific pathogen-free conditions. Mice were housed under standard conditions for a minimum of one week before experimentation. All experiments were performed in accordance with European Community rules and approved by the Animal Care Committee COMETHEA (Comite d'Ethique en Expérimentation Animale du Centre INRA de Jouy-en-Josas et AgroParisTech, Jouy en Josas, France) and the Canadian Council on Animal Care Guidelines (CCAC).

\section{Low grade-inflammation induction and $F$. prausnitzii administration}

The experimental protocol is detailed in Figure 1A. Briefly, mice of approximately $20 \mathrm{~g}$ were fully anesthetized by intraperitoneal (i.p.) injection of $150 \mu \mathrm{l}$ of $0.1 \%$ ketamine (Imalgene 1000, Merial, France) and 0.06\% xylazine (Rompun, Alcyon, France) and a 3.5 catheter (French catheter, Solomon Scientific, France) attached to a tuberculin syringe was inserted into the colon. A dose of $100 \mathrm{mg} / \mathrm{Kg}$ of DNBS solution (ICN, Biomedical Inc.) in $30 \%$ ethanol (EtOH) was then injected intrarectally (i.r.). Control mice (without colitis) received only 30\% $\mathrm{EtOH}$. Ten days after the DNBS acute period (the three days following DNBS administration during which the effects are greatest), $200 \mu \mathrm{l}$ of $1 \mathrm{X} 10^{9} \mathrm{CFU}$ of bacteria in PBS, or PBS alone, or culture supernatant (SN) were administered intragastrically, daily for 10 days (gavage period). The DNBS administration was repeated 21 days after the first DNBS injection (recovery period) with a second injection of $50 \mathrm{mg} / \mathrm{Kg}$ of DNBS solution. Weight loss was monitored during 3 days following the second DNBS injection to assess possible clinical signs of distress.

To confirm the absence of overt inflammation, macroscopic and histological scores as well as myeloperoxidase (MPO) activity were determined as previously described [25].

\section{Lipocalin-2 (Lcn-2) levels}

Before the mice were sacrificed, blood samples were obtained from the retro-orbital venous plexus, and centrifuged for $10 \mathrm{~min}$ at $12,000 \mathrm{rpm}$ and $4^{\circ} \mathrm{C}$. Clear supernatants (serum) were collected and stored at $-20^{\circ} \mathrm{C}$ until analysis. Serum samples were diluted in the kitrecommended diluent (1\% BSA in PBS), and Lcn-2 was assayed using Duoset murine Lcn-2 ELISA kit (R\&D Systems, Minneapolis, MN) [36].

\section{Cytokine assays}

One centimeter samples of distal colon were recovered and homogenized in an appropriate volume (to give $50 \mathrm{mg} / \mathrm{ml}$ ) of Tris- $\mathrm{HCl}$ buffer containing protease inhibitors (Sigma-Aldrich) in a Tissue Lyser (Qiagen). The samples were centrifuged for $20 \mathrm{~min}$ and the supernatants collected and frozen at $-80^{\circ} \mathrm{C}$ until analysis. Blood samples were obtained from the retro-orbital venous plexus before the mice were killed and centrifuged, and the sera stored at $-80^{\circ} \mathrm{C}$ until analysis. IL-6, IL-10, IFN- $\gamma$, TNF- $\alpha$, IL-5, IL-2, IL-22, IL-1 $\alpha$, IL-13, IL-17, IL4, IL-27 and IL-12p70 were assayed with a cytometric bead array system (Mouse Th1/Th2/Th17/Th22 13plex Flowcytomix) (eBioscience, San Diego, USA).

\section{Intestinal permeability in vitro and in vivo}

Three days after the final DNBS challenge, intestinal permeability in vitro was assessed by the Ussing chamber technique as previously described (World Precision Instruments, Sarasota, FL, USA) [56,57]. Two sections of colon from each mouse were assessed. Tissue conductance (the passive permeability to ions) and mucosal-toserosal flux of the paracellular probe ${ }^{51} \mathrm{Cr}$-EDTA were determined to assess barrier function and paracellular permeability. ${ }^{51} \mathrm{Cr}$-EDTA flux was calculated as the average over a 2-h period and is expressed as $\% \mathrm{hot} / \mathrm{cm}^{2} / \mathrm{hr}$.

Permeability in vivo was assessed using fluorosceinconjugated dextran (FITC-dextran 3000-5000 Da, SigmaAldrich) as previously described [58]. Briefly, three days after the second DNBS injection, $0.6 \mathrm{mg} / \mathrm{g}$ body weight of FITC-dextran was administered to mice by oral gavage and $3.5 \mathrm{~h}$ later, blood samples were obtained from the retro-orbital venous plexus. Plasma FITC levels were determined by fluorometry using a microplate reader (Tecan, Lyon, France). 


\section{Apical junctional analysis by quantitative real-time PCR (qPCR) and immunostaining}

Total RNA was isolated from 20-30 mg samples of colon with an RNeasy Mini Kit (Qiagen). Column DNAse treatment (Qiagen) was used to eliminate potential DNA contamination. RNA quantity and quality were checked with a NanoDrop aparatus (Thermo Scientific) and by agarose gel electrophoresis. Only samples with intact RNA were used for subsequent cDNA synthesis with iScript reverse transcriptase (Bio-Rad): $500 \mu \mathrm{g}$ of the total RNA preparation was used for each sample. Quantitative real-time PCR (qPCR) was performed with diluted cDNA (10x) in triplicate and with an iQ5 Real-Time Detection System (Bio-Rad). The reaction mix consisted of Sofast Evagreen Supermix (Bio-Rad), primers at $0.5 \mu \mathrm{M}$ (Additional file 2: Table S1), and $2 \mu \mathrm{L}$ of diluted cDNA. Values are expressed as relative fold differences normalized to a housekeeping gene, Gapdh, by the $2^{-\Delta \Delta C T}$ method. All procedures were performed according to the manufacturers' instructions.

Protein expression of apical junctional proteins was evaluated also using immunofluorescence. Colon samples were embedded in Tissue-Tek OCT (Sakura, Torrance, CA). Frozen sections were then cut $(5 \mu \mathrm{m})$, fixed with 3\% paraformaldehyde (PFA) for 15 minutes at $20^{\circ} \mathrm{C}$, and blocked with phosphate-buffered saline (PBS)/ bovine serum albumin at $2 \%$ for 1 hour. Samples were immune-stained overnight with E-cadherin antibody (1:1000 dilution, BD Pharmaceutical), Occludin (1:200, Invitrogen), Claudin 4 (1:200, Invitrogen), F11r (1:100, R\&D) and 1 hour with appropriate secondary antibody (1:250 dilution, Molecular Probes). Representative pictures from each animal were taken with the same exposure time.

\section{Colonic serotonin assays}

One centimeter-long sections of colon were recovered and homogenized $(50 \mathrm{mg} / \mathrm{ml})$ in PBS buffer containing protease inhibitors and Serotonin Stabilizer (LDN, Nordhon, Germany) in a Tissue Lyser (Qiagen). The colon contents were recovered by washing with $1 \mathrm{ml}$ of PBS buffer containing protease inhibitors and Serotonin Stabilizer (LDN) and centrifuged; the supernatant stored at $-80^{\circ} \mathrm{C}$ until analysis. Serotonin concentrations were determined using a Serotonin Research ELISA (LDN) according to the manufacturer's instructions.

\section{Serotonin transporter (SERT) expression in colon}

Total RNA was extracted from $1 \mathrm{~cm}$-long sections of colon homogenized in a TissueLyser, by the guanidinium thiocyanate method [59]. RNA concentration and purity were determined using a Nanodrop ND-1000 apparatus (Thermo Scientific) and the RNA Integrity Number (RIN) was checked using an Agilent 2100 bioanalyzer
(ICE platform, INRA, Jouy-en-Josas, France) and an RNA 6000 Nano LabChip ${ }^{\star}$ kit (Agilent technologies). Reverse transcription was performed with 0.1 to $5 \mu \mathrm{g}$ of RNA of each sample using the High-Capacity cDNA Archive Kit (Applied Biosystems, France) according to the manufacturer's instructions. The absence of PCR inhibition was confirmed for each cDNA sample with the TaqMan ${ }^{\circledast}$ Exogenous Internal Positive Control (Applied Biosytems, France). The cDNA products were analyzed in triplicate by RT-qPCR with an ABI PRISM 7000 Sequence Detection System and the 7000 system software version 1.2.3 (Applied Biosytems). Several genes were analyzed using TaqMan Gene Expression Assays (Applied Biosystems, France), including Slc6a4 (Mm00439391_m1) and gapdh (Mm99999915_g1), used as a reference. The results obtained were normalized to the value for gapdh rRNA and compared with the mean target gene expression in the ETOH-PBS group as the calibrator sample. The following formula was used: fold change $=2^{-\Delta \Delta \mathrm{Ct}}$, where $\Delta \Delta \mathrm{Ct}$ threshold cycle $(\mathrm{Ct})$ equals (target $\mathrm{Ct}$ - reference $\mathrm{Ct}$ ) of sample minus (target $\mathrm{Ct}$ - reference $\mathrm{Ct}$ ) of the calibrator.

\section{Statistical analysis}

GraphPad software (GraphPad Sofware, La Jolla, CA, USA) was used for statistical analysis. Results are presented as bar graphs or dot plots with means +/- SEM. Comparisons involved the non-parametric KruskalWallis test was used, followed by a Dunn's Multiple Comparison test. A $p$ value of less than 0.05 was considered significant.

\section{Additional files}

Additional file 1: Figure S1. Effect of F. prausnitzii on apical junction proteins in a DNBS-induced low-grade inflammation model. Sections of the distal colon were stained for Claudin-4, Fr11, occludin (green) and E-cadherin (red) expression. Nuclei (DAPI; blue). Original magnification X20. Representative images from control non-inflamed (EtOH-PBS), control inflamed (DNBS-PBS), bacteria-free culture medium (DNBSLYBHI), F. prausnitzii strain A2-165 (DNBS-Fprau), and F. prausnitzii A2-165 SN (DNBS-SN) groups.

Additional file 2: Table S1. Primers used in this study.

\section{Abbreviations}

AJ: Adherens junction proteins; DNBS: Dinitro-benzene sulfonic acid EC: Enterochromaffin cells; EtOH: Ethanol; H\&E: Hematoxylin and eosin; IBD: Inflammatory bowel disease; i.p.: Intraperitoneal; i.r.: Intrarectally; Lcn-2: Lipocalin 72; LYBHI: Brain-heart infusion medium supplemented with $0.5 \%$ yeast extract; MPO: Myeloperoxidase; PFA: Paraformaldehyde; qPCR: Quantitative real-time PCR; 5-HT: Serotonin; SERT: Serotonin reuptake transporter; SN: Supernatant; TJ: Tight junction proteins.

\section{Competing interests}

The authors declare that they have no competing interests.

\section{Authors' contributions}

$R M, E F V, P B, V T, P L$ and $L G B H$ designed all the experiments. RM, SM, FC, $\mathrm{LGBH}, J M N, J L, J J$ and $\mathrm{HS}$ performed the experiments. RM, LGBH and PL wrote the manuscript. SM, FC, EFV, PB and VT corrected the manuscript. All authors read and approved the final manuscript. 


\section{Authors' information}

P. Langella and L. Bermudez share the senior co-authorship.

\section{Acknowledgements}

This study was a part of a FPARIS collaborative project selected and supported by the Vitagora Competitive Cluster and funded by the French FUI (Fond Unique Interministériel; FUl: $n^{\circ}$ F1010012D), the FEDER (Fonds Européen de Développement Régional; Bourgogne: 34606), the Burgundy Region, the Conseil Général 21 and Grand Dijon. This work was also supported by Merck Médication Familiale (Dijon, France) and Biovitis (Saint Etienne de Chomeil, France). RM and SM receive a salary from these same grants. EFV holds a Canada Research Chair. The work was partially funded by CCFC grants to PL, EFV and PB.

We thank Stephen Collins and Muriel Thomas for their advice during this work and Laure Laval and all UEAR personal (especially Mathilde Bauducel) for their technical help. PL thanks S. Collins and John Wallace for his stay as a visiting professor in Farncombe Institute where this work was initiated.

\section{Author details}

${ }^{1}$ INRA, Commensal and Probiotics-Host Interactions Laboratory, UMR 1319 Micalis, F-78350 Jouy-en-Josas, France. ${ }^{2}$ AgroParisTech, UMR1319 Micalis, F-78350 Jouy-en-Josas, France. ${ }^{3}$ Farncombe Family Digestive Health Research Institute, McMaster University, 1200 Main St West, H.Sc. 3N6, Hamilton, Ontario, Canada. ${ }^{4}$ INSERM, Equipe AVENIR U1057 / UMR CNRS 7203, 75012 Paris, France. ${ }^{5}$ Department of Gastroenterology and Nutrition, AP-HP, Hôpital Saint-Antoine F-75012 and UPMC Univ Paris 06F-75005, Paris, France. ${ }^{6}$ INRA, Neuro-Gastroenterology and Nutrition Team, UMR 1331 Toxalim, F-31931 Toulouse, France.

\section{Received: 18 July 2014 Accepted: 2 March 2015} Published online: 21 March 2015

\section{References}

1. Martin R, Miquel S, Ulmer J, Langella P, Bermudez-Humaran LG. Gut ecosystem: how microbes help us. Benef Microbes. 2014;5(3):219-33.

2. Tap J, Mondot S, Levenez F, Pelletier E, Caron C, Furet JP, et al. Towards the human intestinal microbiota phylogenetic core. Environ Microbiol. 2009;11(10):2574-84

3. Sokol H, Lay C, Seksik P, Tannock GW. Analysis of bacterial bowel communities of IBD patients: what has it revealed? Inflamm Bowel Dis. 2008;14(6):858-67.

4. Kostic AD, Xavier RJ, Gevers D. The microbiome in inflammatory bowel disease: current status and the future ahead. Gastroenterology. 2014;146(6):1489-99.

5. Rajilic-Stojanovic M, Biagi E, Heilig HG, Kajander K, Kekkonen RA, Tims S, et al. Global and deep molecular analysis of microbiota signatures in fecal samples from patients with irritable bowel syndrome. Gastroenterology. 2011;141(5):1792-801.

6. Khan S, Chang L. Diagnosis and management of IBS. Nat Rev Gastroenterol Hepatol. 2010;7(10):565-81.

7. Rana SV, Sharma S, Sinha SK, Parsad KK, Malik A, Singh K. Pro-inflammatory and anti-inflammatory cytokine response in diarrhoea-predominant irritable bowel syndrome patients. Trop Gastroenterol. 2012;33(4):251-6.

8. Barbara G, Cremon C, Stanghellini V. Inflammatory bowel disease and irritable bowel syndrome: similarities and differences. Curr Opin Gastroenterol. 2014;30(4):352-8.

9. Collins SM. Is the irritable gut an inflamed gut? Scand J Gastroenterol Suppl. 1992;192:102-5.

10. Peterson J, Garges S, Giovanni M, Mclnnes P, Wang L, Schloss JA, et al. The NIH Human Microbiome Project. Genome Res. 2009;19(12):2317-23.

11. Akiho $H$, Ihara $E$, Nakamura K. Low-grade inflammation plays a pivotal role in gastrointestinal dysfunction in irritable bowel syndrome. World J Gastrointest Pathophysiol. 2010;1(3):97-105.

12. Vivinus-Nebot M, Frin-Mathy G, Bzioueche H, Dainese R, Bernard G, Anty R, et al. Functional bowel symptoms in quiescent inflammatory bowel diseases: role of epithelial barrier disruption and low-grade inflammation. Gut. 2014;63(5):9

13. Halpin SJ, Ford AC. Prevalence of symptoms meeting criteria for irritable bowel syndrome in inflammatory bowel disease: systematic review and meta-analysis. Am J Gastroenterol. 2012;107(10):1474-82.

14. Simren M, Axelsson J, Gillberg R, Abrahamsson H, Svedlund J, Bjornsson ES. Quality of life in inflammatory bowel disease in remission: the impact of
IBS-like symptoms and associated psychological factors. Am J Gastroenterol. 2002;97(2):389-96.

15. Moss AC. The meaning of low-grade inflammation in clinically quiescent inflammatory bowel disease. Curr Opin Gastroenterol. 2014;30(4):365-9.

16. Fries $W$, Belvedere A, Vetrano S. Sealing the broken barrier in IBD: intestinal permeability, epithelial cells and junctions. Curr Drug Targets. 2013;14(12):1460-70.

17. Spiller R, Lam C. The shifting interface between IBS and IBD. Curr Opin Pharmacol. 2011;11(6):586-92.

18. Edelblum $\mathrm{KL}$, Turner JR. The tight junction in inflammatory disease: communication breakdown. Curr Opin Pharmacol. 2009;9(6):715-20.

19. Lee BJ, Bak YT. Irritable bowel syndrome, gut microbiota and probiotics. J Neurogastroenterol Motil. 2011;17(3):252-66.

20. DuPont AW, DuPont HL. The intestinal microbiota and chronic disorders of the gut. Nat Rev Gastroenterol Hepatol. 2011;8(9):523-31.

21. Miquel S, Martin R, Rossi O, Bermudez-Humaran LG, Chatel JM, Sokol H, et al. Faecalibacterium prausnitzii and human intestinal health. Curr Opin Microbiol. 16(3):255-261.

22. Sokol H, Pigneur B, Watterlot L, Lakhdari O, Bermudez-Humaran LG, Gratadoux JJ, et al. Faecalibacterium prausnitzii is an anti-inflammatory commensal bacterium identified by gut microbiota analysis of Crohn disease patients. Proc Natl Acad Sci U S A. 2008;105(43):16731-6.

23. Miquel S, Martin R, Rossi O, Bermudez-Humaran L, Chatel J, Sokol H, et al. Faecalibacterium prausnitzii and human intestinal health. Curr Opin Microbiol. 2014;16(3):255-61.

24. Varela E, Manichanh C, Gallart M, Torrejon A, Borruel N, Casellas F, et al. Colonisation by Faecalibacterium prausnitzii and maintenance of clinical remission in patients with ulcerative colitis. Aliment Pharmacol Ther. 2013;38(2):151-61.

25. Martin R, Chain F, Miquel S, Lu J, Gratadoux JJ, Sokol H, et al. The commensal bacterium faecalibacterium prausnitzii is protective in DNBS-induced chronic moderate and severe colitis models. Inflamm Bowel Dis. 2014;20(3):417-20.

26. Ohman L, Simren M. New insights into the pathogenesis and pathophysiology of irritable bowel syndrome. Dig Liver Dis. 2007;39(3):201-15.

27. Bonaz B. Inflammatory bowel diseases: a dysfunction of brain-gut interactions? Minerva Gastroenterol Dietol. 2013;59(3):241-59.

28. Tornblom H, Abrahamsson H, Barbara G, Hellstrom PM, Lindberg G, Nyhlin $\mathrm{H}$, et al. Inflammation as a cause of functional bowel disorders. Scand J Gastroenterol. 2005:40(10):1140-8.

29. Bercik P, Collins SM, Verdu EF. Microbes and the gut-brain axis. Neurogastroenterol Motil. 2012;24(5):405-13.

30. Bolino CM, Bercik P. Pathogenic factors involved in the development of irritable bowel syndrome: focus on a microbial role. Infect Dis Clin North Am. 2010;24(4):961-75. ix

31. Clarke G, Cryan JF, Dinan TG, Quigley EM. Review article: probiotics for the treatment of irritable bowel syndrome-focus on lactic acid bacteria. Aliment Pharmacol Ther. 2012;35(4):403-13.

32. Wrzosek L, Miquel S, Noordine ML, Bouet S, Joncquel Chevalier-Curt M, Robert $V$, et al. Bacteroides thetaiotaomicron and Faecalibacterium prausnitzii influence the production of mucus glycans and the development of goblet cells in the colonic epithelium of a gnotobiotic model rodent. BMC Biol. 2013;11:61

33. Qin HY, Xiao HT, Wu JC, Berman BM, Sung JJ, Bian ZX. Key factors in developing the trinitrobenzene sulfonic acid-induced post-inflammatory irritable bowel syndrome model in rats. World J Gastroenterol. 2012;18(20):2481-92.

34. Wallace JL, Le T, Carter L, Appleyard CB, Beck PL. Hapten-induced chronic colitis in the rat: alternatives to trinitrobenzene sulfonic acid. J Pharmacol Toxicol Methods. 1995;33(4):237-9.

35. Wang Y, Lam KS, Kraegen EW, Sweeney G, Zhang J, Tso AW, et al. Lipocalin-2 is an inflammatory marker closely associated with obesity, insulin resistance, and hyperglycemia in humans. Clin Chem. 2007;53(1):34-41.

36. Shashidharamurthy R, Machiah D, Aitken JD, Putty K, Srinivasan G, Chassaing $B$, et al. Differential role of lipocalin 2 during immune complex-mediated acute and chronic inflammation in mice. Arthritis Rheum. 2013;65(4):1064-73.

37. Vijay-Kumar M, Wu H, Jones R, Grant G, Babbin B, King TP, et al. Flagellin suppresses epithelial apoptosis and limits disease during enteric infection. Am J Pathol. 2006;169(5):1686-700. 
38. Suzuki T. Regulation of intestinal epithelial permeability by tight junctions. Cellular and molecular life sciences: CMLS. 2013;70(4):631-59.

39. Tian T, Yu S, Ma D. Th22 and related cytokines in inflammatory and autoimmune diseases. Expert Opin Ther Targets. 2013;17(2):113-25.

40. Van Dyken SJ, Locksley RM. Interleukin-4- and interleukin-13-mediated alternatively activated macrophages: roles in homeostasis and disease. Annu Rev Immunol. 2013;31:317-43.

41. Sanchez-Munoz F, Dominguez-Lopez A, Yamamoto-Furusho JK. Role of cytokines in inflammatory bowel disease. World J Gastroenterol. 2008;14(27):4280-8.

42. Luzina IG, Keegan AD, Heller NM, Rook GA, Shea-Donohue T, Atamas SP. Regulation of inflammation by interleukin-4: a review of "alternatives". J Leukoc Biol. 2012;92(4):753-64.

43. Al-Sadi R, Boivin M, Ma T. Mechanism of cytokine modulation of epithelial tight junction barrier. Front Biosci. 2009;14:2765-78.

44. Capaldo CT, Nusrat A. Cytokine regulation of tight junctions. Biochim Biophys Acta. 2009:1788(4):864-71.

45. Kiesslich R, Duckworth CA, Moussata D, Gloeckner A, Lim LG, Goetz M, et al. Local barrier dysfunction identified by confocal laser endomicroscopy predicts relapse in inflammatory bowel disease. Gut. 2012;61(8):1146-53.

46. Carlsson AH, Yakymenko O, Olivier I, Hakansson F, Postma E, Keita AV, et al. Faecalibacterium prausnitzii supernatant improves intestinal barrier function in mice DSS colitis. Scand J Gastroenterol. 2013;48(10):1136-44.

47. Talley NJ. Irritable bowel syndrome. Intern Med J. 2006;36(11):724-8.

48. Coates MD, Johnson AC, Greenwood-Van Meerveld B, Mawe GM. Effects of serotonin transporter inhibition on gastrointestinal motility and colonic sensitivity in the mouse. Neurogastroenterol Motil. 2006;18(6):464-71.

49. Khan WI, Ghia JE. Gut hormones: emerging role in immune activation and inflammation. Clin Exp Immunol. 2010;161(1):19-27.

50. Bertrand PP, Barajas-Espinosa A, Neshat S, Bertrand RL, Lomax AE. Analysis of real-time serotonin (5-HT) availability during experimental colitis in mouse. Am J Physiol Gastrointest Liver Physiol. 2010;298(3):G446-55.

51. Clarke G, Quigley EM, Cryan JF, Dinan TG. Irritable bowel syndrome: towards biomarker identification. Trends Mol Med. 2009;15(10):478-89.

52. Spiller R. Serotonin and GI clinical disorders. Neuropharmacology. 2008;55(6):1072-80

53. Foley KF, Pantano C, Ciolino A, Mawe GM. IFN-gamma and TNF-alpha decrease serotonin transporter function and expression in Caco2 cells. Am J Physiol Gastrointest Liver Physiol. 2007:292(3):G779-84.

54. Linden DR, Foley KF, McQuoid C, Simpson J, Sharkey KA, Mawe GM. Serotonin transporter function and expression are reduced in mice with TNBS-induced colitis. Neurogastroenterol Motil. 2005;17(4):565-74.

55. Urdaci MC, Sanchez B. Some immunomodulatory effects of probiotic bacteria might be due to porcine neutrophil elastase inhibitor, a serpin present in MRS broth. Immunol Lett. 2009;122(1):99-100.

56. Galipeau HJ, Rulli NE, Jury J, Huang X, Araya R, Murray JA, et al. Sensitization to gliadin induces moderate enteropathy and insulitis in nonobese diabetic-DQ8 mice. J Immunol. 2011;187(8):4338-46.

57. Natividad JM, Huang X, Slack E, Jury J, Sanz Y, David C, et al. Host responses to intestinal microbial antigens in gluten-sensitive mice. PLoS One. 2009;4(7):e6472.

58. Tambuwala MM, Cummins EP, Lenihan CR, Kiss J, Stauch M, Scholz CC, et al. Loss of prolyl hydroxylase-1 protects against colitis through reduced epithelial cell apoptosis and increased barrier function. Gastroenterology. 2010:139(6):2093-101.

59. Chomczynski P, Sacchi N. Single-step method of RNA isolation by acid guanidinium thiocyanate-phenol-chloroform extraction. Anal Biochem. 1987;162(1):156-9.

\section{Submit your next manuscript to BioMed Central and take full advantage of:}

- Convenient online submission

- Thorough peer review

- No space constraints or color figure charges

- Immediate publication on acceptance

- Inclusion in PubMed, CAS, Scopus and Google Scholar

- Research which is freely available for redistribution

Submit your manuscript at www.biomedcentral.com/submit 